\title{
Characterization of Specific Noncovalent Complexes between Guanidinium Derivatives and Single-Stranded DNA by MALDI
}

\author{
Keiichiro Ohara, Michael Smietana, and Jean-Jacques Vasseur \\ Laboratoire de Chimie Organique Biomoléculaire de Synthèse, CNRS-Université Montpellier II, Montpellier, \\ France
}

\begin{abstract}
Noncovalently bound complexes between highly basic sites of 12 guanidinium compounds and single-stranded DNA were studied using matrix-assisted laser desorption/ionization (MALDI) mass spectrometry. 6-Aza-2-thiothymine (ATT) was used as the matrix in the presence of ammonium citrate, and spectra were recorded in the positive ion mode. Detailed control experiments confirmed unambiguously the high selectivity and specificity of the guanidinium moiety for phosphate groups of DNA. The results verify the binding stoichiometry and show preferential binding of hydrophobic binders (pyrene and anthracene guanidinium derivatives) to all sequences examined. In addition, we demonstrate that electrostatic noncovalent interactions are strengthened with phosphorothioate analogs of DNA. These results clearly highlight the structure-directing role of the self-assembling organic species and strongly emphasize the significance of concentration, hydrophobicity, hydrogen-bonding, and $\pi-\pi$ interactions of the artificial receptor in the formation of these noncovalent complexes. Because of the ability of DNA-binding compounds to influence gene expression, and therefore cell proliferation and differentiation, the interactions described above could be important in providing a better understanding of the mechanism of action of these noncovalent genetic regulators. (J Am Soc Mass Spectrom 2006, 17, 283-291) (c 2006 American Society for Mass Spectrometry
\end{abstract}

$\mathrm{T}$ The function of a biomolecule usually depends on its specific, noncovalent interactions with another molecule such as hydrogen-bonding (H-bonding), ion-dipole or hydrophobic interactions. These interactions drive critically important cellular processes such as drug action, cell signalling, ion transport, and energy transfer. Interest in the use of noncovalent binding interactions involving the cationic guanidinium group has grown significantly in recent years [1]. This moiety interacts strongly with anionic groups such as carboxylates, phosphates, and sulfates through noncovalent hydrogen bonding and charge-pairing interactions. The formation of these noncovalent complexes can essentially be explained by the high pKa (around 12-13) of the guanidinium group, which is consequently protonated over a wide $\mathrm{pH}$ range. Also, the shape complementarity where the guanidinium group aligns with the anionic species leads to very stable noncovalent structures [2]. In this context, arginine residues have been shown to mediate numerous biological noncovalent interactions, including protein/metal, protein/protein,

Published online January 27, 2006

Address reprint requests to Dr. J.-J. Vasseur, Laboratoire de Chimie Organique Biomoléculaire de Synthèse, U.M.R. 5625 CNRS-UMII, Case Courrier 008, Université Montpellier II, Place Eugène Bataillon 34095 Montpellier Cedex 5, France. E-mail: vasseur@univ-montp2.fr protein/peptide, and protein/oligonucleotide [1, 3]. Ongoing efforts to improve our understanding of cellular processes at the molecular level are enhanced by new or improved methods for deriving information about those noncovalent interactions. In particular, obtaining binding stoichiometry and specificity and elucidating the structure of these complexes are of primary importance.

Mass spectrometrists have taken an active role in investigating noncovalent complexes involving biopolymers, and many examples have been published on the use of ESI for their detection [4, 5]. Matrix-assisted laser desorption/ionization (MALDI), another soft ionization method, has also been successfully used in this area of research. Independent work from Biemann and colleagues [6] and Vertes and coworkers [7] showed that MALDI was a viable alternative to investigate noncovalent interactions between highly acidic negatively charged compounds such as heparins or oligonucleotides and basic residues such as arginine-rich peptides. In 1995, Woods et al. detected enzyme-substrate complexes and suggested that the effect of an organic acid matrix on the tertiary structure of proteins and peptides, rather than the laser desorption/ionization process itself, is the primary limitation to the observation of noncovalent complexes in MALDI [8]. That same year, Lecchi and Pannell reported that using 6-aza-2-thiothymine (ATT) as the matrix in the 
presence of ammonium citrate allowed the detection of intact double-stranded DNA [9]. ATT was then chosen by Woods et al. for the detection of peptide-peptide and peptide-oligonucleotide noncovalent interactions [10-12]. Recently, extensive work has been done by Zenobi and coworkers toward the unambiguous detection of noncovalent complexes by MALDI. Specific zinc finger peptideoligonucleotide complexes [13, 14] and DNA duplexes were reported [15]. They also analyzed noncovalent complexes between proteins and sulfonate receptors and were able to probe the number of accessible arginine sites on the surface of folded peptides and proteins [16-19]. Following these results, they used guanidinium derivatives to detect carboxylic residues of peptides [18]. Surprisingly, in contrast to the theory, none of the expected noncovalent complexes between guanidinium groups and carboxylic acid residues were detected by MALDI.

In the past few years, our group showed that attaching cationic amino and guanidinium groups to the backbone of DNA is one way to achieve efficient antisense and antigene oligonucleotides formed with single-stranded and double-stranded nucleic acids, respectively [20, 21]. The increased duplex and triplex stabilities observed were attributed to a reduced overall negative charge of the modified complexes and by the formation of strong salt bridges between basic cationic groups of the oligonucleotide and acidic phosphodiester linkages of the nucleic acid target. In light of the importance of these interactions in gene regulation, the objective of this study was to identify the structural features that drive guanidinium-oligonucleotide complex formation.

Numerous efforts have been devoted to the development of receptors for anionic species. This has been especially true for guanidinium-based variations that have been incorporated into synthetic molecules to create selective receptors and ligands [22, 23]. It has already been shown that the use of a pyrene-functionalized monoguanidinium receptor was a valuable method for specific recognition of the biologically relevant pyrophosphate (PPi) [24]. The binding of the receptor to PPi in a 2:1 ratio was monitored by fluorescence measurements of the characteristic excimer band at $476 \mathrm{~nm}$. Unfortunately, the complex could only be visualized in methanol at a fairly high concentration $\left(8.0 \times 10^{-4} \mathrm{M}\right)$. In light of these results, the goals of the experiments presented here are 3-fold: (1) to determine whether and how MALDI could be used to characterize noncovalent interaction between guanidinium probes and single-stranded DNA; (2) to identify the structural elements that drive these interactions; (3) to confirm the binding stoichiometry and specificity. For this purpose, a series of guanidinium derivatives was synthesized. The results of our experiments demonstrate that DNA/ guanidinium complexes are transferred in a nondestructive way into the gas-phase, and we will discuss the structural requirements for successful detection of these complexes.

\section{Experimental}

\section{Mass Spectrometry}

MALDI-TOF mass spectra were recorded on a Voyager DE (PerSeptive Biosystems, Framingham, MA) mass spectrometer equipped with an $\mathrm{N}_{2}$ laser $(337 \mathrm{~nm}$ ). MALDI conditions were as follows: laser power, 25002700 (arbitrary units); all spectra shown in this paper have been acquired in positive linear mode with an acceleration voltage of $24 \mathrm{kV}$; guide wire, $0.05 \%$ of accelerating voltage; grid voltage, $94 \%$ of accelerating voltage; delay extraction time, $550 \mathrm{~ns}$. All spectra reported here were not smoothed. Typically, 40-50 laser shots were averaged for each spectrum.

\section{Sample Preparation}

6-Aza-2-thiothymine (ATT) and ammonium citrate were purchased from Aldrich (Saint Quentin Fallavier, France) and used without further purification. Fresh matrix solution was prepared daily by adding $1.7 \mathrm{mg}$ of ammonium citrate to $200 \mu \mathrm{L}$ of a saturated of solution of ATT in acetonitrile/water (1:1 (vol/vol), HPLC grade). All the analytes solutions were prepared in deionized water with concentrations of $10^{-4} \mathrm{M}$. All the guanidinium solutions were prepared in deionized water or in methanol with concentrations of $10^{-3}$ to $3 \times$ $10^{-3} \mathrm{M}$. The analyte and guanidinium solutions were mixed with the matrix solution (1:1:4) in a total volume of $2.5 \mu \mathrm{L}$. The mixture was deposited on the MALDI plate and left to dry slowly at room-temperature. When the effect of salt concentration was studied, the guanidinium/oligonucleotide mixture was further incubated with $\mathrm{NaCl}$ in a 1:1 (vol/vol) ratio for $5 \mathrm{~min}$.

\section{Materials}

Oligonucleotides were synthesized on an Applied Biosystems Model 381A (ABI, Foster City, CA) DNA synthesizer using protocols and reagents for standard phosphoramidite chemistry [25]. A solution of $3 \mathrm{H}$ 1,2-benzodithiol-3-one $0.05 \mathrm{M}$ in acetonitrile was used as an oxidizer for the formation of thionophosphotriester internucleoside linkages [26]. Methyl phosphonate sequences were synthesized following the standard methylphosphonate chemistry [27]. Guanidinium hydrochloride, agmatine sulfate, and arginine were obtained commercially (Aldrich). All other guanidinium derivatives shown in Table 1 were prepared by guanylation of the corresponding amines [28] and obtained as hydrochloride salts. Their structures were confirmed by ${ }^{1} \mathrm{H}$ and ${ }^{13} \mathrm{C}$ NMR and HRMS (high resolution mass spectrometry). All compounds used in this study are shown in Table 1. 2,4,6-Trihydroxyacetophenone (THAP), 6-aza-2-thiothymine (ATT), 3-hydroxypicolinic acid (HPA), and nicotinic acid (NA) were all purchased from Aldrich. 
Table 1. Cationic guanidinium receptors investigated in this study. Nominal masses in brackets correspond to the protonated form

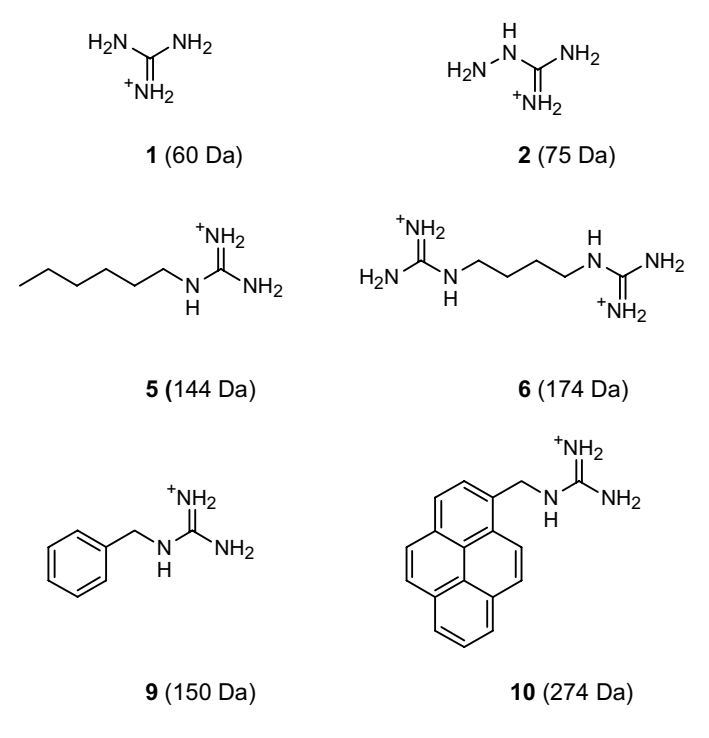

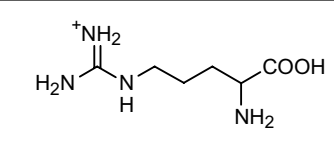

$3(175 \mathrm{Da})$<smiles>NC(=[NH2+])NC1CCCCC1</smiles>

7 (142 Da)

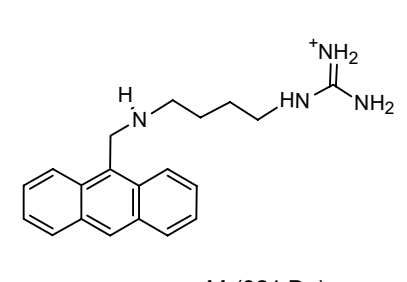

11 (321 Da)<smiles>NCCCCNC(N)N</smiles>

4 (131 Da)<smiles>NC([NH3+])NCc1cccs1</smiles>

8 (156 Da)

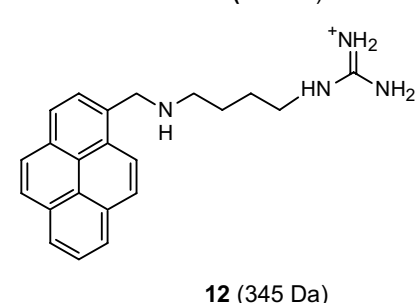

\section{Results and Discussion}

It is well known that the principal guanidinium-phosphate interaction is a salt bridge increased by iondipole interactions and hydrogen bonding. To explore the nature of the interactions involved, we investigated the effect of modifications to the guanidinium structure as well as the influence of nucleotide base and DNA backbone composition. The impact of these factors was deduced from the number and abundance of noncovalently bound complexes observed by MALDI. Among all matrix types tested (HPA, THAP, NA, ATT), ATT with ammonium citrate as a comatrix was found to give the best results in the positive mode. Although some complexes could be seen in the negative mode, their intensities as well as the number of the complexes formed were much lower. In the discussion below, we use the notation introduced by Juhasz and Biemann [6]. That is, $(m: n)^{+}$describes the composition and charge state of the complex ion $\left[\mathrm{mM}_{\mathrm{ODN}}+\mathrm{nM}_{\mathrm{GUA}}+\mathrm{H}\right]^{+}$, where $\mathrm{M}_{\mathrm{ODN}}$ and $\mathrm{M}_{\mathrm{GUA}}$ refer to the molecular weight of the oligonucleotide and the guanidinium derivative, respectively. The oligonucleotide sequences studied and the molecular weights of their monomers are listed in Table 2.

To determine if the structure of the guanidylated molecule plays an active role in this ionic complexation, 12 structurally distinct guanidinium derivatives were tested. Nonspecific aggregation is always a concern in the study of noncovalent association. With this in mind, we evaluated the influence of varying guanidinium solution concentration upon the formation and detection of noncovalent complexes with various analytes. In some cases, the number of adducts would depend on the relative molar ratio between analyte and guanidinium species. By probing a 10- to 30-fold molecular excess of guanidinium while keeping the analyte concentration constant at $10^{-4} \mathrm{M}$, more complexes could be seen along with an increase in their respective intensities, but no adduct signal over the maximum guanidinium/phosphate stoichiometry was ever observed. The effect of molar ratios on the observation of complex formation is illustrated in Figure 1, where guanidinium probe 9 and Sequence 1 were used. By using a 30-fold molecular excess of the probe, ions up to three adducts

Table 2. Oligonucleotides used in the non-covalent binding experiments

\begin{tabular}{|c|c|c|}
\hline Oligonucleotide & Sequence & $\mathrm{m} / \mathrm{z}$ (calc.) \\
\hline 1 & 5'-CGATCG-3' & 1792.2 \\
\hline 2 & $5^{\prime}-\mathrm{AAAA}-3^{\prime}$ & 1190.9 \\
\hline 3 & 5'-TTTTTTTTTTTT-3' & 3588.4 \\
\hline 4 & 5'-AGAATTGGGTGT-3' & 3740.5 \\
\hline 5 & 5'-ACACCCAATTCT-3' & 3549.4 \\
\hline 6 & 5'-TTTTTTT-3' & 2067.4 \\
\hline 7 & $5^{\prime}-T_{p s} T_{p s} T_{p s} T_{p s} T_{p s} T_{p s} T-3^{\prime}$ & 2163.8 \\
\hline 8 & $5^{\prime}-A_{m p} C_{m p} A_{m p} C_{m p} C_{m p} C_{m p} A_{m p} A_{m p} T_{m p} T_{m p} C_{m p}{ }^{T}-3^{\prime}$ & 3527.6 \\
\hline 9 & $5^{\prime}-\mathrm{TA}_{m p} \mathrm{C}_{m p} A_{m p} \mathrm{C}_{m p} \mathrm{C}_{m p} \mathrm{C}_{m p} \mathrm{~A}_{m p} \mathrm{~A}_{m p} \mathrm{~T}_{m p} \mathrm{~T}_{m p} \mathrm{C}_{m p} \mathrm{~T}^{-3^{\prime}}$ & 3831.8 \\
\hline
\end{tabular}

$\mathrm{m} / \mathrm{z}$ refers to theoretical masses of the sequences studied in the positive ion mode. $\mathrm{N}_{\mathrm{ps}}$ corresponds to a nucleotide phosphorothioate analogue and $\mathrm{N}_{\mathrm{mp}}$ to a methylphosphonate analog. 
(a)

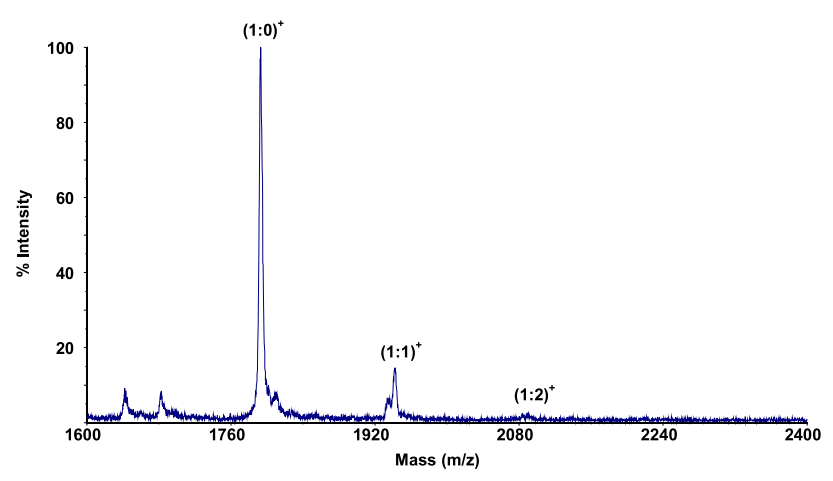

(b)

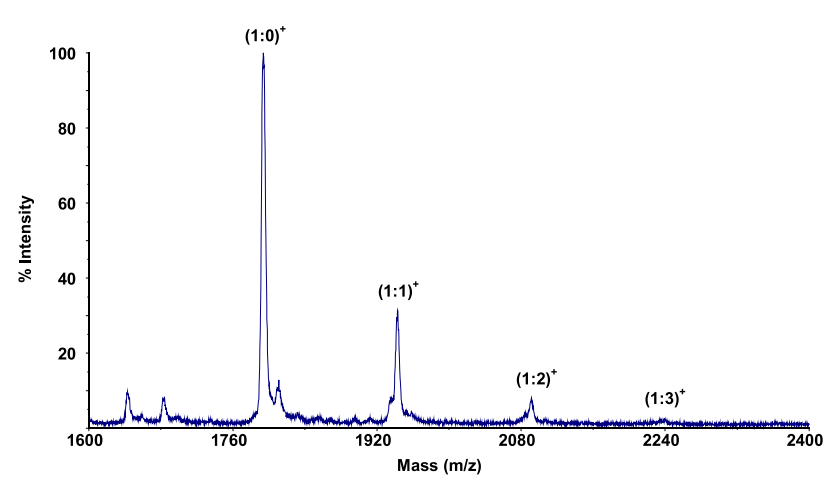

(c)

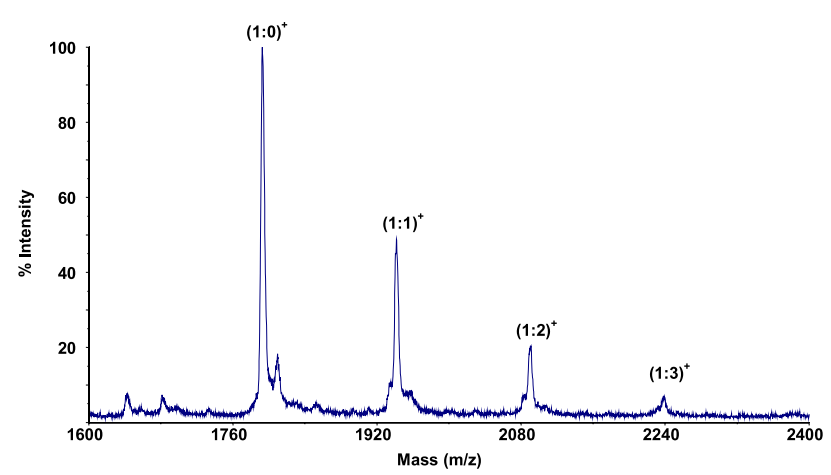

Figure 1. Positive ion mode MALDI mass spectra of Sequence 1 (5'-CGATCG-3') with (a) 10 equivalents; (b) 20 equivalents; (c) 30 equivalents of guanidinium derivative 9. (Small peaks between $\mathrm{m} / \mathrm{z}$ 1600-1750 correspond to Sequence 1 residual impurities).

are detected with a tiny $(1: 3)^{+}$peak. However, with 10 equivalents of probe, only the $(1: 1)^{+}$peak is readily observed, albeit at a very low abundance, while the $(1: 2)^{+}$can only be presumed to exist. In the following experiments, we employ a 10-fold molar excess of guanidinium over the analyte to find the strongestbinding probes.

Our next series of experiments was done on all guanidinium derivatives with Sequence 1. To our delight, most of these guanidinium derivatives formed (a)

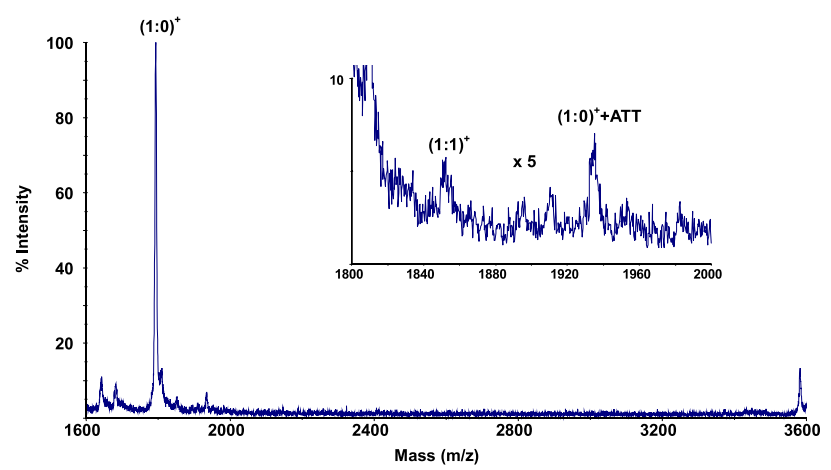

(b)

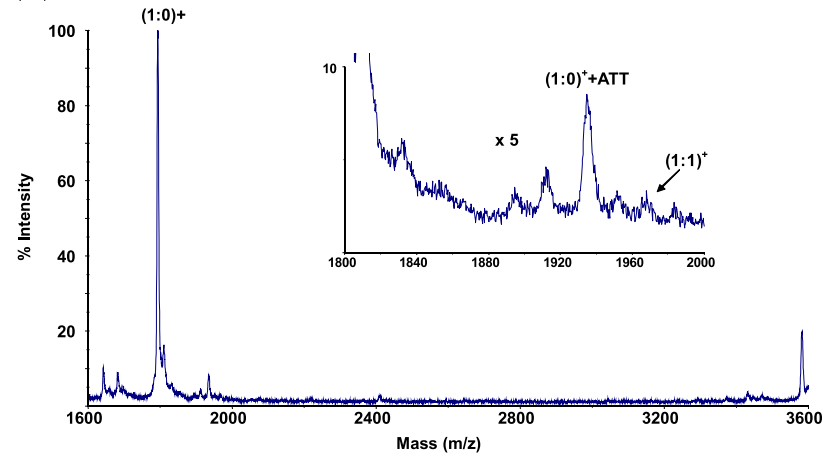

(c)

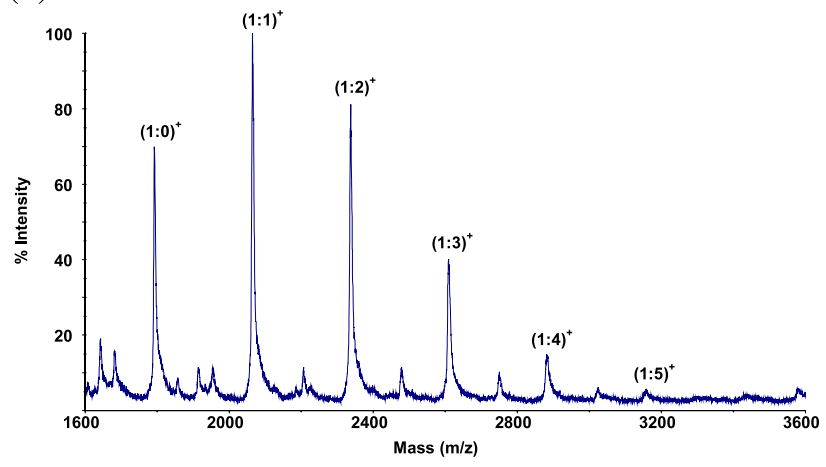

(d)

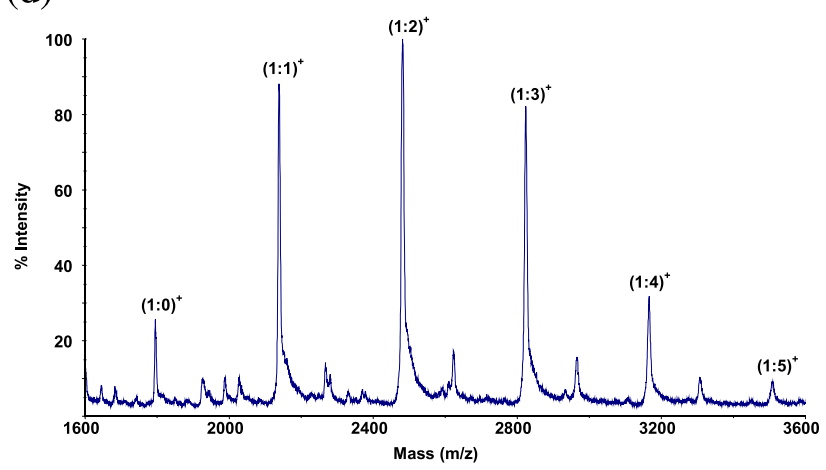

Figure 2. Positive ion mode MALDI mass spectra of Sequence 1 (5'-CGATCG-3') with 10 equivalents of guanidinium derivatives (a) 1; (b) 3; (c) 10; and (d) 12 . 


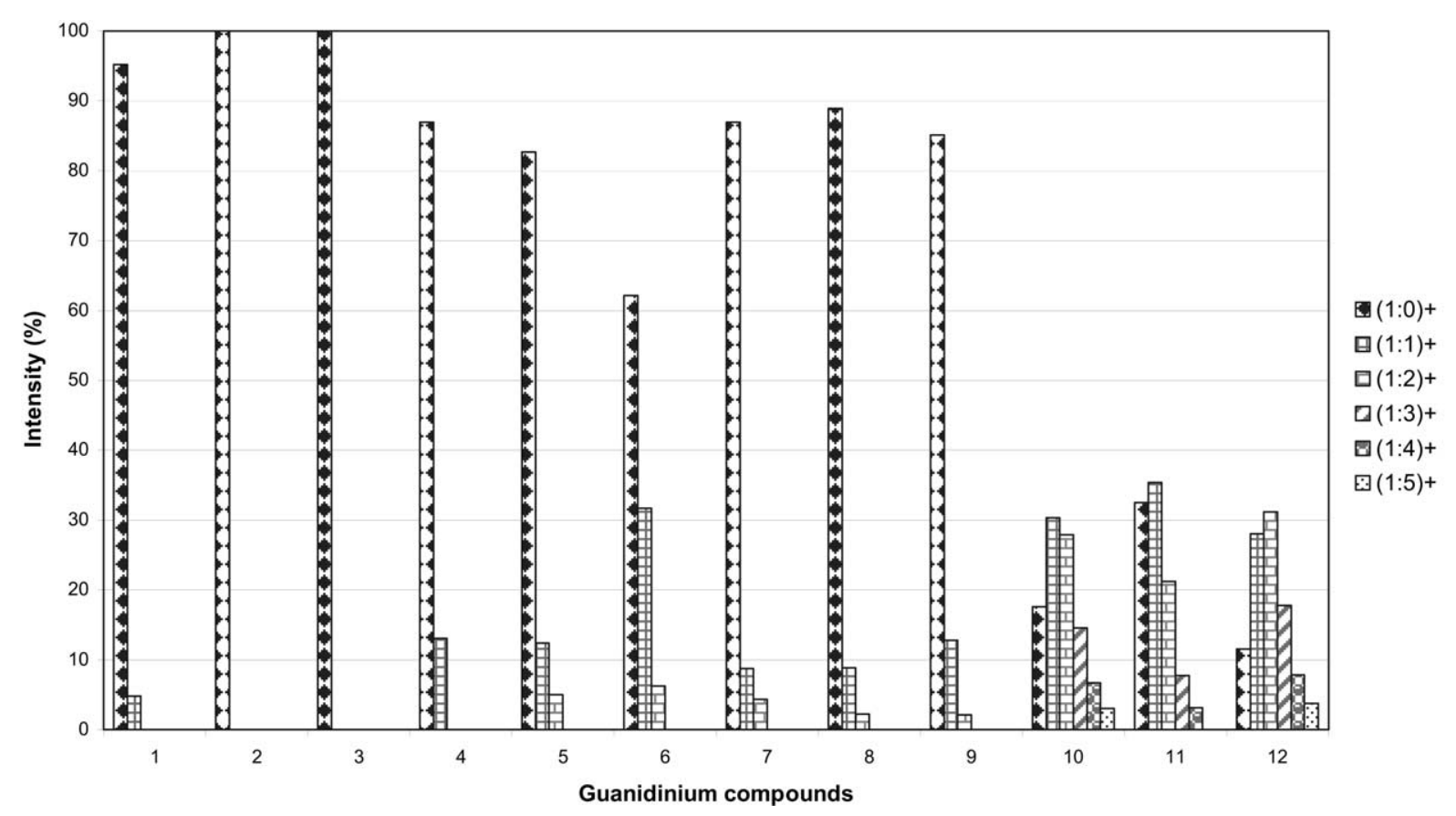

Figure 3. Average intensity ratios of 10 equivalents of guanidinium Compounds 1-12 with Sequence 1.

noncovalent complexes. However, the abundance of complex ions changed considerably depending on the guanidinium structure of the probe. An illustrative example is shown in Figure 2, where Compounds $\mathbf{1}$ (Figure 2a), 3 (Figure 2b), 10 (Figure 2c), and 12 (Figure 2d) are compared. Compound 1 (Figure 2a, inset) exhibits an overriding $(1: 0)^{+}$noncomplexed ion with a slight $(1: 1)^{+}$peak. With arginine 3 (Figure $2 b$, inset) and as a trend with all hydrophilic compounds (Figure 3), only the free oligonucleotide could be observed. A very different profile is observed for Compounds 10 (Figure $2 \mathrm{c}$ ) and 12 (Figure 2d). In those cases, $(1: 0)^{+}$is no longer the most abundant ion. Instead, a ladder of peaks displaying all possible complexes could be observed with $(1: 1)^{+}$and $(1: 2)^{+}$complex ions as the most intense peaks. Moreover, $(1: 3)^{+}$and $(1: 4)^{+}$complex could easily be detected, and $(1: 5)^{+}$complex ion can still be observed, but at a very low intensity. All peaks are separated by the anticipated mass differences of 273 and $344 \mathrm{Da}$, respectively. Nonspecific ATT adducts could also been seen in the spectra. It is also noteworthy that the length of the alkyl chain as a guanidinium modification does not seem to influence the binding ratio. The average relative bindings of all guanidinium compounds with Sequence 1 are represented in Figure 3 and reveal large differences in affinity. Because we could not acquire the entire charge distribution for each species, the abundance ratio for detected ions was used to evaluate their binding affinity. It was found that the abundance ratio of complexes increased dramatically with increasing hydrophobicity of the group attached to the guanidinium. In fact, $(1: 1)^{+},(1: 2)^{+}$and $(1: 3)^{+}$ions start to appear when an alkyl chain is connected to the guanidinium scaffold. Strikingly, $(1: 4)^{+}$, and to a lesser extent, $(1: 5)^{+}$complexes only become visible with the inclusion of large, delocalized aromatic structures (Compounds 10-12). Since these species show a clear preference for binding with oligonucleotides, a higher degree of stabilization from $\pi$-stacking interactions could be assumed. As expected, no complexes were detected with lysine, benzylamine, or pyrene methylamine, all of which are less able to form noncovalent bonds (data not shown). This can easily be explained by the insufficient basicity exhibited by amines to stabilize salt-bridge interactions, at least in the gas phase. As a consequence of these results, we decided for our future experiments to use probe 10, as it showed a high binding affinity for the oligonucleotide phosphate linkage and is the most easily synthesized probe.

The influence of salt concentration was investigated next using Sequence 1 . When $\mathrm{NaCl}$ concentration was increased, sodium ions would compete with guanidinium ions in the formation of ionic bonds. Thus, when Sequence 1 and 10 equivalents of Compound 9 were mixed, a total loss of noncovalent complex was noted after incubation with $0.8 \mathrm{M} \mathrm{NaCl}$ (data not shown). Interestingly, even a saturated $\mathrm{NaCl}$ solution was not sufficient to induce total disappearance of complexes with Compound 10. In this case, along with sodium adducts of the free oligonucleotide, sodium adducts of $(1: 1)^{+}$complex ions $(1: 1+\mathrm{Na})^{+},(1: 1+2$ $\mathrm{Na})^{+}$, and even $(1: 1+3 \mathrm{Na})^{+}$were easily detected, demonstrating the unexpected strength of the interactions between hydrophobic guanidinium derivatives and phosphate groups. To a lesser extent, a similar distribution is observed with the $(1: 2)^{+}$ion (Figure 4 ). 


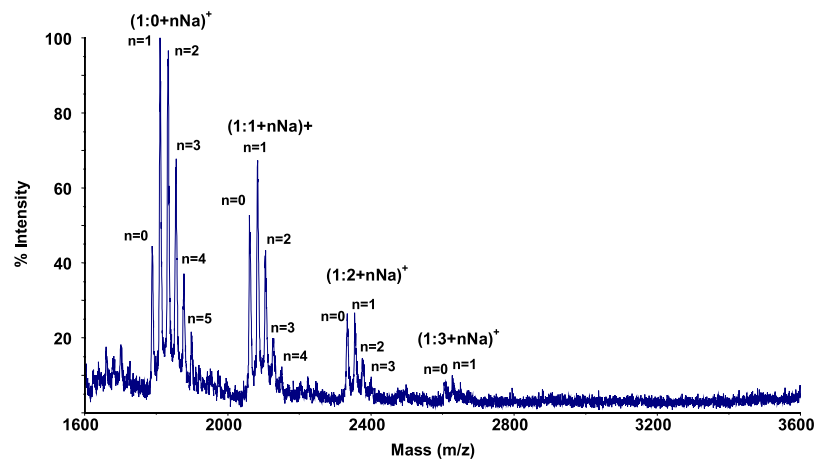

Figure 4. Positive ion mode MALDI mass spectra of Sequence 1 with 10 equivalents of guanidinium derivative $\mathbf{1 0}$ in saturated $\mathrm{NaCl}$.

Compounds $\mathbf{1 1}$ and $\mathbf{1 2}$ led to the same results (data not shown). These observations not only prove the electrostatic nature of the observed complexes but also corroborate the binding potency of hydrophobic probes already observed at low ionic strength. Finally, to prove that DNA/guanidinium noncovalent complexes are preformed in solution and transferred into the gasphase as intact entities, Friess and Zenobi's dry mixing control experiment was performed [17]. In this experiment, Sequence $1\left(10^{-4} \mathrm{M}\right)$ and Compound $10\left(10^{-3} \mathrm{M}\right)$ are dissolved in matrix solution and dried separately. The resulting powder is then mixed without any solvent and deposited on a double-sided adhesive tape. Upon laser irradiation, only $(1: 0)^{+}$ion was spotted. However, after addition of $2 \mu \mathrm{L}$ of a mixture of acetonitrile/water (1:1), the adhesive support was partially dissolved, resulting in the appearance of a large number of peaks in the MALDI mass spectrum. Methanol and even water did not allow us to avoid this partial solubility of the adhesive. We then tried to mix the powder directly on the plate. In this case, $(1: 0)^{+}$ion could still be detected at any position on the powder surface, and no complexes could be identified. After addition of the acetonitrile/water mixture to the plate, all complexes previously seen could be detected, although at a different molecular ratio (data not shown). This control experiment gives evidence that the com-

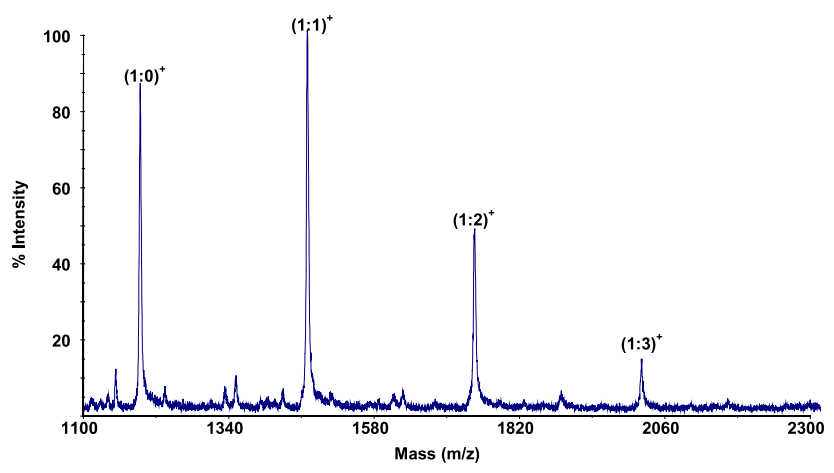

Figure 5. Positive ion mode MALDI mass spectra of Sequence 2 $\left(5^{\prime}\right.$-AAAA- $\left.3^{\prime}\right)$ with 10 equivalents of guanidinium derivative $\mathbf{1 0}$.
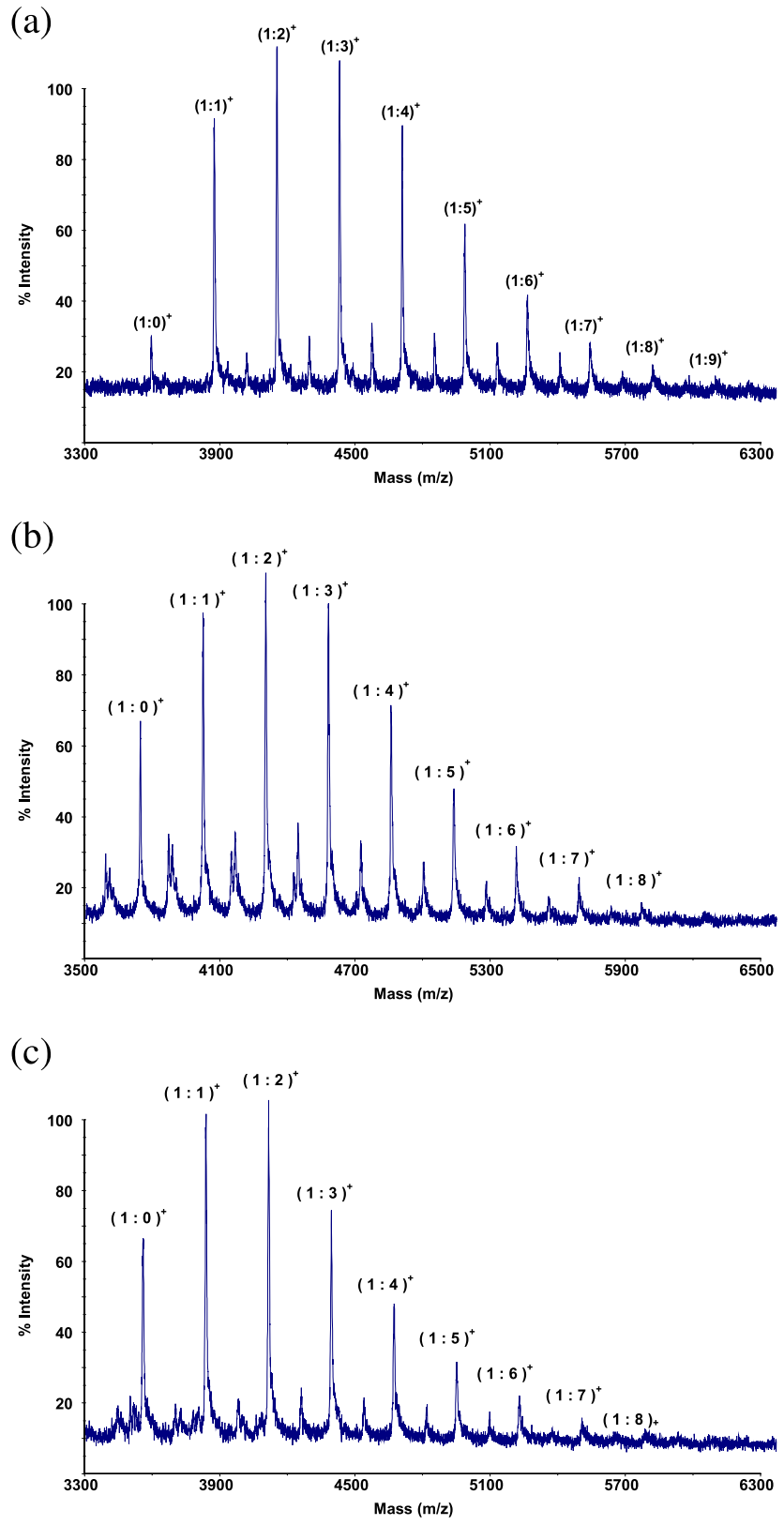

(d)

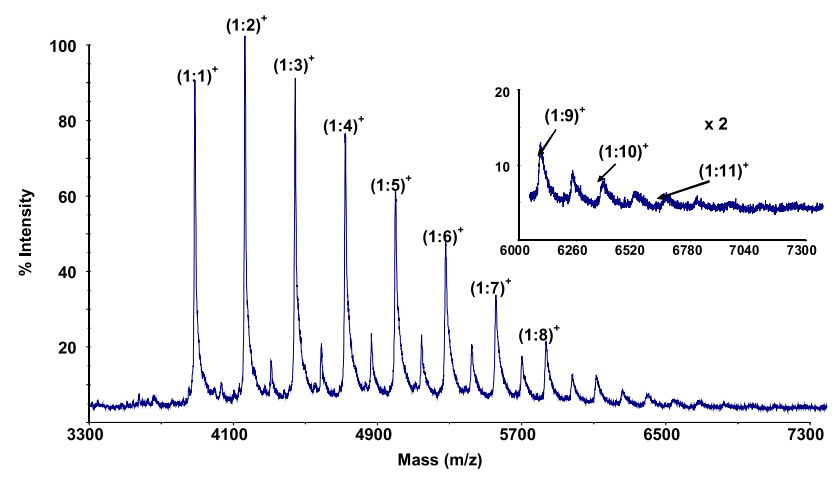

Figure 6. Positive ion mode MALDI mass spectra of 12 mers (a) Sequence 3; (b) Sequence 4; (c) Sequence 5 with 10 equivalents of guanidinium derivative 10; (d) Sequence 3 with 60 equivalents of guanidinium derivative $\mathbf{1 0 .}$ 


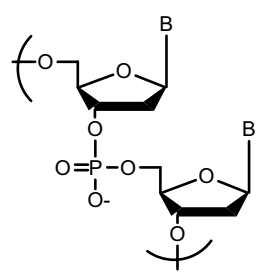

phosphodiester

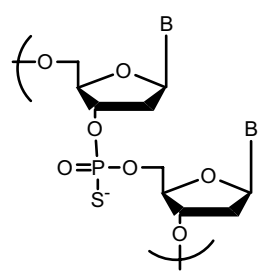

phosphorothioate

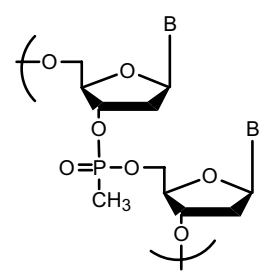

methylphosphonate

Scheme 1. Graphical representation of the different phosphate backbones used in this study.

plexes are not the result of nonspecific gas-phase adduct formation but are instead preformed in solution.

To definitively rule out nonspecific complexation, a shorter sequence containing only three internucleotide linkages was used. Logically, no $(1: 4)^{+}$ion was detected when 10 equivalents of Compound $\mathbf{1 0}$ were mixed with Sequence 2 (Figure 5). Under these conditions, a dominant $(1: 1)^{+}$ion complex was observed along with $(1: 2)^{+}$ and the stoichiometric $(1: 3)^{+}$species, demonstrating selective complexation. With both Sequences 1 and 2, all of the acidic phosphate anions can be complexed with guanidinium groups, but no further complexation beyond the original stoichiometry is seen, and all analytes show a distribution of anion adducts up to the maximum possible number if highly basic cations are added.

To study the influence of the sequence length and the contribution of nucleic acid composition, Sequences 3-5 were used. These 12-mers containing 11 internucleotide linkages were chosen to allow the formation of a large number of complexes. As represented in Figure 6a, 10 equivalents of probe $\mathbf{1 0}$ led to large amounts of $(1: 1)^{+}$ through $(1: 6)^{+}$complex ions and a much lower abundance of $(1: 7)^{+}$and $(1: 8)^{+}$ions. Similar complex distributions were acquired with heteromers (Figure $6 \mathrm{~b}$ and c) demonstrating the absence of base sequence effects on complex formation. Sixty equivalents of probe $\mathbf{1 0}$ were needed to observe extensive complexes-up to eleven units-with homothymidine Sequence 3 . The need for a larger amount of guanidinium compound is evident if one considers the overwhelming abundance of $(1: 1)^{+}$to $(1: 6)^{+}$complex ions. With this condition, even a noticeable amount of the startling $(1: 10)^{+}$and $(1: 11)^{+}$ions could be detected (Figure 6d, inset).

We then decided to direct our studies toward phosphorothioate oligonucleotides. Phosphorothioate analogs in which a nonbridging oxygen atom in the phosphodiester linkage has been replaced by a sulfur atom have widespread use in molecular biology (Scheme 1) [29]. The increased resistance of phosphorothioates against nuclease digestion has prompted the consideration of these molecules for medical purposes as antisense probes [30]. In addition to their value, phosphorothioates have also been used to predict site-specific interactions with cations [31], and are important in the investigation of stereospecific interactions in enzymatic systems [32, 33]. It is well known that in phosphorothioate anions the $\mathrm{P}-\mathrm{S}$ bond is a single bond with a negative charge localized on sulfur [34]. We thus expected a stronger electrostatic character of the phosphorothioate/guanidinium complexes accompanied by less hydrogen bonding, since sulfur and oxygen differ considerably in their ability to form hydrogen bonds [35]. Comparing Sequences 6 and 7 allowed us to prove that electrostatic interaction appears to be a more significant parameter for noncovalent complex formation as compared to hydrogen bonding. Whereas a normal charge distribution was obtained with Sequence 6, almost no $(1: 0)^{+}$ion was observed with Compound 10 (Figure 7)

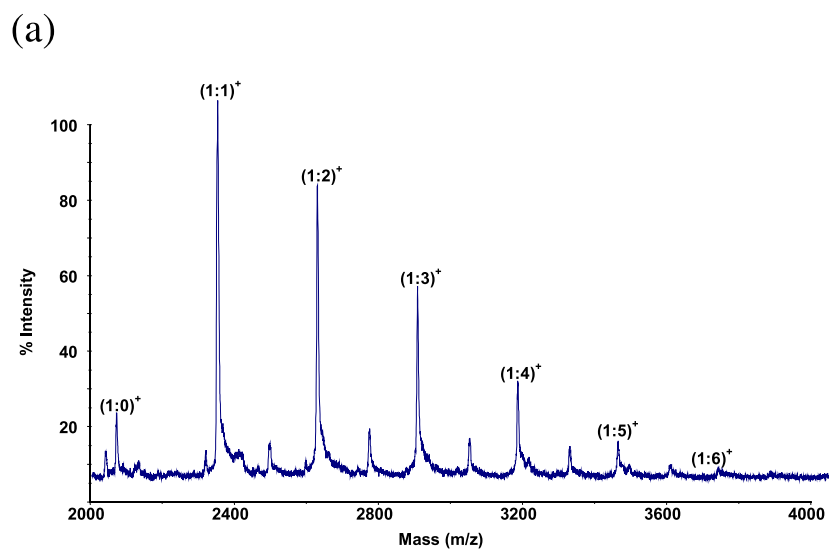

(b)

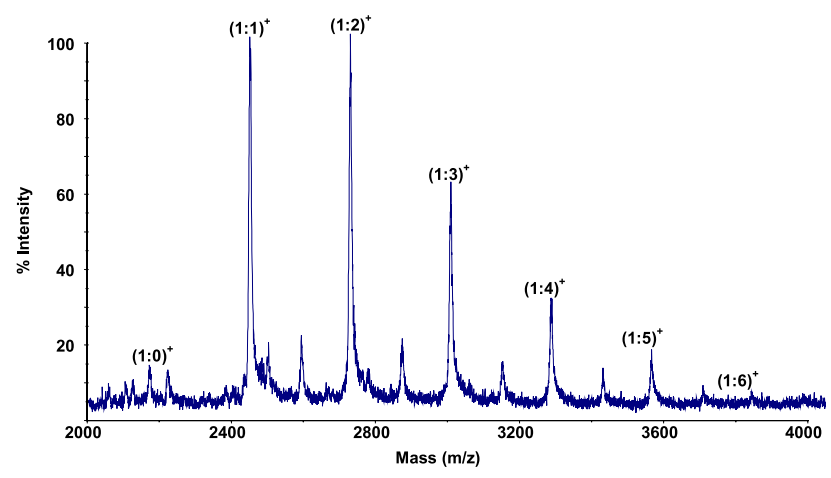

Figure 7. Positive ion mode MALDI mass spectra of 10 equivalents of guanidinium derivative 10 with (a) Sequence 6 and (b) Sequence 7. 


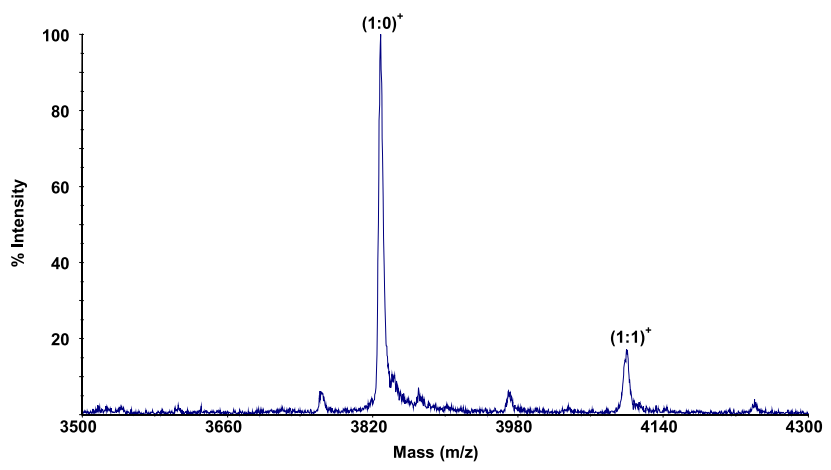

Figure 8. Positive ion mode MALDI mass spectra of Sequence 9 with 10 equivalents of guanidinium derivative $\mathbf{1 0}$.

and Sequence 7. The distribution goes up to the maximum possible number of adducts with $(1: 1)^{+}$and $(1: 2)^{+}$ complexes as the most intense peaks. Furthermore, the full $(1: 6)^{+}$complex is easily observed in both cases.

All the results presented above for single-stranded DNA prompted us to use the high degree of specificity displayed by this method to probe a modified methylphosphonate oligonucleotide. Methylphosphonates are nonionic nucleic acid analogs that contain nucleaseresistant methylphosphonate linkages instead of the naturally occurring negatively charged phosphodiester bonds (Scheme 1). These analogs are hydrophobic and have been found to be effective antisense reagents, capable of specifically controlling viral or cellular gene expression at the mRNA level [36]. This backbone was proposed as an advantageous chemistry by Miller and T'so and colleagues in the 1980s [37]. When the phosphate is blocked as in the structurally-similar methylphosphonate analytes, neither electrostatic nor hydrogen-bonding-type interactions can take place. Since there is only a $2 \mathrm{Da}$ difference between the natural phosphate and its methylphosphonate analog, it might sometimes be difficult to evaluate precisely the number of phosphodiester linkages in a methylphosphonate oligonucleotide. We thus decided to use our guanidinium compounds to probe this number. For this purpose, the spectra of Sequence 8, an all-modified methylphosphonate oligonucleotide was compared with Sequence 9, which bears one standard phosphodiester linkage whereas all others are methylphosphonate ones. Sequences 8 and 9 were examined following our standard conditions (10 equivalents of probe 10). With Sequence 8 , the expected $(1: 0)^{+}$ion was observed along with a tiny amount of $(1: 1)^{+}$ion (less than $4 \%$; data not shown). If we cannot completely rule out nonspecific interactions, we would rather assume that hydrolysis of $4 \%$ of the methylphosphonate linkage was the origin of the $(1: 1)^{+}$ion. In the case of Sequence 9 , its structure was undoubtedly corroborated when a $(1: 1)^{+}$complex ion was visualized along with fair amount of the anticipated (1:0) ${ }^{+}$peak (Figure 8 ). In this experiment, no other complexes were observed even with 80 equivalents of probe 10, but at that concentration the ratio of
$(1: 0)^{+}$over $(1: 1)^{+}$ion goes up to $2 / 1$. This methodology can therefore be extended to all other nonionic modifications of the DNA backbone and could theoretically be used to probe the degree of hydrolysis of a modified oligonucleotide.

\section{Conclusions}

Interactions between nucleic acids and proteins play an important role in numerous biochemical processes, including DNA replication, recombination and repair. Arginine residues are significant determinants of the nature of these biomolecular associations, notably by noncovalently interacting with a variety of other amino acids or by intermolecularly mediating complexation with phosphate groups. This behavior is usually attributed in large part to the positive charge on the guanidinium group. This study revealed that the structural characteristics that drive formation of specific noncovalent complexes between guanidinium species and various analytes are influenced significantly by hydrophobic and $\pi$-stacking interactions. In conclusion, the occurrence and strength of a guanidinium-phosphate complex in a single strand of DNA depends on: (1) the concentration of the artificial receptor, (2) its hydrophobicity, and (3) its ability to form $\pi-\pi$ interactions. Moreover, the complexation specificity demonstrated here allowed us to probe the degree of modification of a methylphosphonate analog of DNA. This methodology could therefore be applied to all other types of inter nucleosidic linkage modifications that meet the minimum requirements of guanidinium binding. Owing to the dynamic nature of the self-assembled structures shown here, it is anticipated that similar DNA binders with high binding affinities could be able to interfere with gene expression.

\section{Acknowledgments}

The authors are grateful to Andrea Kurtz for careful reading of the manuscript and helpful comments. This work was funded in part by the CNRS and Université Montpellier II. Dr. F. Debart is acknowledged for generously providing Sequences 8 and 9.

\section{References}

1. Schug, K. A.; Lindner, W. Noncovalent Binding Between Guanidinium and Anionic Groups: Focus on Biological- and Synthetic-Based Arginine/Guanidinium Interactions with Phosph[on]ate and Sulf[on]ate Residues. Chem. Rev. 2005, 105, 67-113.

2. Calnan, B. J.; Tidor, B.; Biancalana, S.; Hudson, D.; Frankel, A. D. Arginine-Mediated RNA Recognition: The Arginine Fork. Science 1991, 252, 1167-1171.

3. Brooks, H.; Lebleu, B.; Vives, E. Tat Peptide-Mediated Cellular Delivery: Back to Basics. Adv. Drug Delivery Rev. 2005, 57, 559-577.

4. Hofstadler, S. A.; Griffey, R. H. Analysis of Noncovalent Complexes of DNA and RNA by Mass Spectrometry. Chem. Rev. 2001, 101, 377-390.

5. Loo, J. A. Studying Noncovalent Protein Complexes by Electrospray Ionization Mass Spectrometry. Mass Spectrom. Rev. 1997, 16, 1-23.

6. Juhasz, P.; Biemann, K. Mass Spectrometric Molecular Weight Determination of Highly Acidic Compounds of Biological Significance via their Complexes with Basic Polypeptides. Proc. Natl. Acad. Sci. U.S.A. 1994, 91, 4333-4337.

7. Tang, X.; Callahan, J. H.; Zhou, P.; Vertes, A. Noncovalent Protein Oligonucleotide interactions Monitored by Matrix-Assisted Laser De- 
sorption/Ionization Mass Spectrometry. Anal. Chem. 1995, 67, 4542-4548.

8. Woods, A. S.; Buchsbaum, J. C.; Worrall, T. A.; Berg, J. M.; Cotter, R. J. Matrix-Assisted Laser Desorption/Ionization of Noncovalently Bound Compounds. Anal. Chem. 1995, 67, 4462-4465.

9. Lecchi, P.; Pannell, L. K. The Detection of Intact Double-Stranded DNA by MALDI. J. Am. Soc. Mass Spectrom. 1995, 6, 972-975.

10. Lin, S.; Long, S.; Ramirez, S. M.; Cotter, R. J.; Woods, A. S. Characterization of the "Helix Clamp" Motif of HIV-1 Reverse Transcriptase Using MALDI-TOF MS and Surface Plasmon Resonance. Anal. Chem. 2000, 72, 2635-2640.

11. Lin, S.; Cotter, R. J.; Woods, A. S. Detection of noncovalent interaction of single and double stranded DNA with peptides by MALDI-TOF. Proteins. 1998, Suppl. 2, S12-S21.

12. Woods, A. S.; Huestis, M. A. A Study of Peptide-Peptide Interaction by Matrix-Assisted Laser Desorption/Ionization. J. Am. Soc. Mass Spectrom. 2001, 12, 88-96

13. Lehmann, E.; Zenobi, R. Detection of Specific Noncovalent Zinc Finger Peptide- Oligodeoxynucleotide Complexes by Matrix-Assisted Laser Desorption/Ionization Mass Spectrometry. Angew. Chem. Int. Ed. 1998, 37, 3430-3432

14. Lehmann E.; Zenobi, R.; Vetter, S. Matrix-Assisted Laser Desorption/ Ionization Mass Spectra Reflect Solution-Phase Zinc Finger Peptide Complexation. J. Am. Soc. Mass Spectrom. 1999, 10, 27-34.

15. Sudha, R.; Zenobi, R. The Detection and Stability of DNA Duplexes Probed by MALDI Mass Spectrometry. Helv. Chim. Acta 2002, 85, 3136-3143

16. Salih, B.; Zenobi, R. MALDI Mass Spectrometry of Dye-Peptide and Dye-Protein Complexes. Anal. Chem. 1998, 70,1536-1543.

17. Friess, S. D.; Zenobi, R. Protein Structure Information from Mass Spectrometry? Selective Titration of Arginine Residues by Sulfonates. J. Am. Soc. Mass Spectrom. 2001, 12, 810-818.

18. Friess, S. D.; Jürg, M. D.; Hartmann, R.; Zenobi, R. Mass Spectrometric Noncovalent probing of Amino Acids in Peptides and Proteins. Int. J. Mass Spectrom. 2002, 219, 269-281.

19. Friess, S. D.; Jürg, M. D.; Zenobi, R. Probing the Surface Accessibility of Proteins with Noncovalent Receptors and MALDI Mass Spectrometry. Phys. Chem. Chem. Phys. 2004, 6, 2664-2675.

20. Michel, T.; Martinand-Mari, C.; Debart, F.; Lebleu, B.; Robbins, I.; Vasseur, J.-J. Cationic Phosphoramidate $\alpha$-Oligonucleotides Efficiently Target Single-stranded DNA and RNA and Inhibit Hepatitis C Virus IRES-Mediated Translation. Nucleic Acids Res. 2003, 31, 5282-5290

21. Michel, T.; Debart, F.; Heitz, F.; Vasseur, J.-J. Highly Stable DNA Triplexes Formed with Cationic Phosphoramidate Pyrimidine $\alpha$-Oligonucleotides. ChemBioChem. 2005, 6, 1254-1262.

22. Schmidtchen, F. P.; Berger, M. Artificial Organic Host Molecules for Anions. Chem. Rev. 1997, 97, 1609-1646.

23. Best, M. D.; Tobey, S. L.; Anslyn, E. V. Abiotic Guanidinium Containing Receptors for Anionic Species. Coord. Chem. Rev. 2003, 240, 3-15.
24. Nishizawa, S.; Kato, Y.; Teramae, N. Fluorescence Sensing of Anions via Intramolecular Excimer Formation in a Pyrophosphate-Induced SelfAssembly of a Pyrene-Functionalized Guanidinium Receptor. J. Am. Chem. Soc. 1999, 121, 9463-9464.

25. Beaucage, S. L.; Caruthers, M. H. Synthetic Strategies and Parameters Involved in the Oligodeoxyribonucleotides According to the Phosphoramidite Method. Curr. Prot. Nucleic Acids 2000, 3.3.1-3.3.20.

26. Iyer, R. P.; Egan, W.; Regan, J. B.; Beaucage, S. L. 3H-1,2-Benzodithiole3-One 1,1 Dioxide as an Improved Sulfurizing Reagent in the SolidPhase Synthesis of Oligodeoxyribonucleoside Phosphorothioates. J. Am. Chem. Soc. 1990, 112, 1253-1254.

27. Hogrefe, R. I.; Vaghefi, M. M.; Reynolds, M. A.; Young, K. M.; Arnold, J. J. Deprotection of Methylphosphonate Oligonucleotides Using a Novel One-Pot Procedure. Nucleic Acids Res. 1993, 21, 2031-2038.

28. Pons, J. F.; Fauchereb, J. L.; Lamaty, F.; Mollac, A.; Lazaro, R. A Constrained Diketopiperazine as a New Scaffold for the Synthesis of Peptidomimetics. Eur. J. Org. Chem. 1998, 853-859.

29. Eckstein, F.; Gish, G. Phosphorothioates in Molecular Biology. Trends Biochem. Sci. 1989, 14, 97-100.

30. Cazenave, C.; Helene, C. Antisense Oligonucleotides. In Antisense Nucleic Acids and Proteins, Fundamentals and Applications, Mol, J.N.; Van der Krol, A.R., Eds.; Marcel Dekker: New York, 1991; pp 47-93.

31. Pecoraro, V. L.; Hermes, J. D.; Cleland, W. W. Stability Constants of $\mathrm{Mg}^{2+}$ and $\mathrm{Cd}^{2+}$ Complexes of Adenine Nucleotides and Thionucleotides and Rate Constants for Formation and Dissociation of MgATP and MgADP. Biochemistry 1984, 22, 5262-5271.

32. Zhao, L.; Liu, Y.; Bruzik, K. S.; Tsai, M.-D. A Novel Calcium-Dependent Bacterial Phosphatidylinositol-Specific Phospholipase C Displaying Unprecedented Magnitudes of Thio Effect, Inverse Thio Effect, and Stereoselectivity. J. Am. Chem. Soc. 2003, 125, 22-23.

33. Kravchuk, A. V.; Zhao, L.; Kubiak, R. J.; Bruzik, K. S.; Tsai, M.-D. Mechanism of Phosphatidylinositol-Specific Phospholipase C: Origin of Unusually High Nonbridging Thio Effects. Biochemistry 2001, 40, 54335439.

34. Frey, P. A.; Sammons R. D. Bond Order and Charge Localization in Nucleoside Phosphorothioates. Science 1985, 228, 541-545.

35. Frey, P. A. Chiral Phosphorothioate-Stereochemical Analysis of Enzymatic Substitution at Phosphorus. Adv. Enzymol. Relat. Areas Mol. Biol. 1989, 62, 119-201.

36. Reynolds, M. A.; Arnold, L. J., Jr.; Almazon, M. T.; Beck, T. A.; Hogrefe, R. I.; Metzler, M. D.; Stoughton, S. R.; Tseng, B. Y.; Trapane, T. L.; Ts'o, P. O. P.; Woolf, T. M. Triple-Strand Forming Methylphosphonate Oligodeoxynucleotides Targeted to mRNA Efficiently Block Protein Synthesis. Proc. Natl. Acad. Sci. U.S.A. 1994, 91, 12433-12437.

37. Miller, P. S.; McParland, K. B.; Jayaraman, K.; Ts'o, P. O. P. Biochemical and Biological Effects of Nonionic Nucleic Acid Methylphosphonates. Biochemistry 1981, 20, 1874-1880. 